Published in final edited form as:

Clin J Pain. 2015 May ; 31(5): 414-424. doi:10.1097/AJP.0000000000000124.

\title{
Opioid Use in the Management of Diabetic Peripheral Neuropathy (DPN) in a Large Commercially Insured Population
}

Pravinkumar R. Patil, B.S.Pharm., M.A., M.S., M.B.A., R.Ph. ${ }^{1}$, Jonathan Wolfe, Ph.D., R.Ph. ${ }^{2}$, Qayyim Said, Ph.D. ${ }^{1}$, Jeremy Thomas, Pharm.D. ${ }^{2}$, and Bradley C. Martin, Pharm.D., Ph.D.

${ }^{1}$ Division of Pharmaceutical Evaluation and Policy, UAMS, Little Rock, AR 72205

2University of Arkansas for Medical Sciences, College of Pharmacy, Little Rock, AR, 72205

\begin{abstract}
Objective-To examine the proportion of diabetic peripheral neuropathy (DPN) patients receiving pharmacologic DPN treatments and specifically to identify the rates and factors associated with opioid use and first line opioid use.
\end{abstract}

Methods-A 10\% sample of IMS-LifeLink claims data from 1998 through 2008 was used. The study population consisted of diabetic patients who met DPN criteria using a validated DPN algorithm. Multivariable logistic regression controlling for demographics, comorbidities, and other clinical characteristics was used to identify factors associated with any DPN pharmacologic treatment, any opioid use, and first-line opioid treatment. Sensitivity analyses were conducted to explore variations in exclusion criteria as well as opioid use definitions.

Results-666 DPN patients met inclusion criteria and pharmacologic treatment was received by 288 subjects (43.24\%) and of those, 154 (53.47\%) had DPN related opioid use and 96 (33.33\%) received opioid as first line treatment. Persons with diabetic complications were more likely to use opioids (OR=4.53, 95\% CI=1.09-18.92). FDA approved DPN agents duloxetine 1.04\% $(\mathrm{n}=3)$ and pregabalin $5.56 \%(\mathrm{n}=16)$ had much lower rates of use. DPN related drug use and DPN related opioid usage increased as we used less restrictive samples in sensitivity analyses.

Conclusion-Opioids were the most frequently prescribed first line agents for DPN. More than $50 \%$ of DPN patients remained untreated with pharmacologic agents one year after a DPN diagnosis.

\section{Keywords}

DPN=Diabetic peripheral neuropathy; NCPCs=Non-cancer pain conditions; NSAIDs=Nonsteroidal anti-inflammatory drugs; Opioids

*Corresponding Author: Bradley C Martin, Pharm.D., Ph.D., Professor and Head, Division of Pharmaceutical Evaluation and Policy, University of Arkansas for Medical Sciences College of Pharmacy, 4301 W. Markham, slot 522, Little Rock, AR 72205,

BMartin@uams.edu, 501.603.1992; 501.686 .8315 (fax). 


\section{Introduction}

Diabetes is the leading cause of morbidity and is the $5^{\text {th }}$ leading cause of death in the United States. ${ }^{1}$ Diabetic complications are the major cause of mortality and increases healthcare cost in the United States. ${ }^{2}$ Diabetic neuropathy (DN) is a common complication of diabetes mellitus, caused by decreased blood flow and hyperglycemia. Almost $66 \%$ of type I and $59 \%$ of type II patients suffer from DN and $20 \%$ of all diabetic patients suffer from chronic neuropathic pain (NP) ${ }^{3-4}$ According to the International Association for the Study of Pain ${ }^{4}$ (IASP), Diabetic peripheral neuropathy (DPN) is defined as "pain initiated or caused by a primary lesion or dysfunction in the nervous system". The pain associated with DPN is often described as burning, stabbing, tingling, numbness or itching, and is typically worse at night. Untreated or poorly managed DPN often leads to foot ulceration and lower extremity amputations (LEA) ${ }^{5-6}$ DPN is responsible for impaired health related quality of life, mental and physical well-being. About $12.5 \%$ of DPN patients remain unreported after diagnosis and $39 \%$ do not receive any treatment for DPN. ${ }^{7}$

Current pharmacotherapy for DPN includes tricyclic antidepressants (TCAs), anticonvulsants, non-steroidal anti-inflammatory drugs (NSAIDs), cyclooxygenase 2 inhibitors (COX 2) inhibitors, antiarrhythmics, benzodiazepines, and opioids. ${ }^{8-9}$ Only duloxetine, a selective serotonin norepinephrine reuptake inhibitor (SSNRI), and pregabalin, an anticonvulsant, are currently approved by the FDA for treating DPN. ${ }^{10}$ Currently none of the neuropathy treatment guidelines recommend opioid as an initial choice for patients with DPN. ${ }^{11-16}$ All recommend antidepressants as first-line and anticonvulsants as first or second line agents to treat neuropathic pain. Tramadol and opioids are generally recommended for moderate to severe pain as third line agents.

Use of opioids in NCPCs has been studied ${ }^{17-19}$, but limited information is available about DPN and opioid usage. Currently it is not known how widely opioids are used to treat DPN in a large representative population, nor do we have an understanding of the factors associated with the decision to initiate opioids. ${ }^{20-21}$

To our knowledge, no published studies have studied DPN/neuropathy guidelines concordance with regard to opioid use. The goal of this study was to study the proportion of DPN patients who are prescribed pharmacologic treatments with a focus on opioid use and to determine the proportion of DPN patients who are prescribed opioids as first line treatment. The factors associated with any or first line opioid uses in DPN were also studied. This study further sought to describe the opioid use patterns and describes the duration and dose pattern of opioids (for schedule II long-acting, schedule II short-acting, schedule III-IV short-acting and combinations of different opioid types), and the average daily dose expressed in morphine equivalents.

\section{Methods}

\section{Study design and data source}

A10\% random sample of IMS LifeLink Health Plan claims data from 1998-2008 was analyzed using a retrospective longitudinal study design. The study data and study 
procedures were approved by institutional review board of the University of Arkansas for Medical Sciences. The LifeLink database is comprised of commercial health plan information obtained from over 98 managed care plans and represents the commercially insured population of the United States. This database is representative of the national commercially insured population in terms of age, gender, and geographic location.

\section{Identification of the study sample}

We identified DPN patients based on an ICD-9-CM diagnosis based validated DPN identification algorithm developed by Hartsfield et al. ${ }^{22}$ This ICD-9-CM algorithm was validated with patient chart information and the reported sensitivity, specificity, positive predictive value (PPV), and negative predictive value (NPV) were 94\%, 55\%, 64\% and $92 \%$, respectively, indicating that it was capable of detecting most patients with DPN. Using the algorithm, diabetic patients were first identified based on the presence of one or more primary or secondary ICD-9 CM codes for diabetes $(250.0 \times \mathrm{s}-250.5 \times$ and $250.7 \times-$ $250.9 \times$ ) and/or had received an antidiabetic agent (Appendix 1,Supplemental Digital Content 1, http://links.lww.com/CJP/A118) identified between January 1, 1998 through December $31^{\text {st }}, 2008$. Once diabetic patients were identified, we used the algorithm described in Figure 1 to identify DPN patients. The index diagnosis was the first diagnosis of DPN (ICD-9-CM 250.6, 337.1, 337.9, 354.9, 355.8, 356.9 and 357.2) for persons that met the DPN inclusion criteria described in figure 1.

Once DPN patients were identified the following additional inclusion criteria were applied to ensure persons were eligible and accessing their pharmacy benefits, were adults, and were ambulatory. Patients had to be age $\geq 18$ years, have continuous plan and pharmacy benefit enrollment in the pre- and post- 12 month index periods, no nursing home or hospice care claims, and had at least 1 prescription claim in the post-index period. According to the initial study plan, we were to exclude patients who had opioid exposure prior to their index diagnosis. However, we discovered that many patients initiated DPN pharmacologic treatments before their first DPN diagnosis. Considering this finding, we chose to include patients with prior opioid and other DPN pharmacologic treatments therapy in the pre-index period and chose to exclude persons who had a diagnosis for an indication for DPN pharmacologic treatments (depression, bipolar, epilepsy and anxiety, cancer or painful condition) at any time in our primary analysis. The pain conditions considered were cancer (excluding non-melanoma skin cancer), surgery, pain (headache/migraine, neck pain, back pain, abdominal pain, kidney stone, pelvic pain, rheumatoid arthritis, fracture/contusions and temperomandibular joint pain, and arthritis/joint pain (Appendix 2,Supplemental Digital Content 2, http://links.lww.com/CJP/A119).

\section{Study measures}

Data on demographic and plan characteristic (age, gender, geographic location, insurance payer and product type) were recorded from the plan enrollment file. Clinical characteristics of the DPN patients based on ICD-9-CM diagnosis codes were abstracted from the medical claims file for the pre and post-index 12 month DPN diagnosis period. To measure overall medical comorbidity, the Charlson comorbidity index was used. ${ }^{23}$ To get a measure of noncancer pain conditions in the sensitivity analyses that included these patients (see below), we 
counted the number of unique pain types persons had; headache/migraine, neck pain, back pain, abdominal pain, kidney stone, pelvic pain, rheumatoid arthritis, fracture/contusions, temperomandibular and arthritis/joint pain were used. Mental health and substance use disorders were recorded using ICD-9-CM codes developed by validated grouping software developed by the Agency for Healthcare Research and Quality (AHRQ). ${ }^{24}$ A count of the number of types of metal health conditions (adjustment, depression, anxiety, mood, personality, and miscellaneous disorders) was created and used in the regression models of the sensitivity analysis cohorts. The most frequent type of physician seen by each patient was categorized into four categories; GP/FP/IM (general practitioner / family practitioner / internal medicine), endocrinologists, podiatrists, and other prescribers based on the most frequent prescriber observed in the prescription claims. For persons prescribed DPN drugs, this was the most frequent prescriber type for DPN drugs and those not prescribed DPN drugs the most frequent prescriber of all other prescription claims.

Opioids, antidepressants, anticonvulsants, NSAIDs, topical agents and skeletal muscle relaxants were considered DPN treatments and data on these were obtained from the pharmacy claims file and identified based on GPI codes (Appendix 3, Supplemental Digital Content 3, http://links.lww.com/CJP/A120). Since these drugs have indications for conditions other than DPN and there is no linkage between a drug and its primary use in the data source, we developed a schema to classify those drugs as "DPN Related" if they met the following rules developed by the research team including a clinical pharmacist (Figure 2). If there are no DPN drug prescriptions in the pre-index (365 days) period, then the DPN drug is presumed to be used for DPN and was classified as "DPN related drug use". If the first DPN drug exposure was in the 120 day period prior to the index DPN diagnosis AND the patient had at least one prescription claim for any DPN drug in the post-index 365 days period, then that drug was presumed to treat DPN. The 120 day pre-diagnosis window was based on the premise that DPN drug treatments may be initiated to manage pain prior to a clinical encounter in which DPN is diagnosed. If the first prescribed DPN drug in the 365 day period prior to the index diagnosis, and that same drug is also prescribed in the 120 day period prior to the index diagnosis, and the patient also had at least one prescription claim for any DPN drug/s in the post-index 365 days period, then that drug was presumed to treat DPN. The first drug to meet the definition of DPN related was considered first line use. Use of these drugs that did not meet any of the DPN related definitions was presumed not to treat DPN and was considered 'non-DPN related'.

We describe the DPN drug use that occurred in the post-index 12 months as post-index DPN related drug use. The sum of pre-index DPN related and post-index DPN related drugs was described as total DPN related drug use, and "any use" included DPN related and nonrelated use. DPN patients with no DPN drug use in the pre and post-index 12 month period are characterized as DPN patients with no pharmacologic treatment.

DPN treatment initiation time was calculated as the difference between the date of the first line DPN drug prescription and the index DPN diagnosis date for those who initiated therapy after their DPN diagnosis. For those that initiated DPN treatment prior to the DPN index date, a time to initiation was not calculated. Because opioid use was of specific interest to this study, we report exclusive use of opioids if opioids were the only DPN 
drug(s) prescribed in the pre and post-index 12 month period. Chronic opioid use is defined as more than 90 days of opioid use with less than 32 days gap between the last date of an early prescription and the fill date of the next prescription. If there were multiple prescriptions on the same fill date, we used the maximum value of days' supply for that fill date. We calculated the cumulative and average daily opioid dose in morphine equivalent (Meq) based on opioid usage. ${ }^{25}$ Meq was calculated by multiplying the quantity of each prescription by the opioid strength of the prescription and multiplying this product by a morphine equivalent conversion factor. If quantity was given and days' supply was missing for a prescription, Meq was calculated for that prescription but average daily dose was not calculated.

\section{Analysis}

For all study cohorts we obtained and reported the baseline descriptive characteristics for DPN treatment receivers, no treatment receivers and any opioid receivers. Multivariable logistic regression models were estimated to explore factors associated with DPN pharmacologic treatment, any DPN related opioid use, first line opioid and chronic opioid use. These models were estimated hierarchically, where the entire sample was used to identify factors associated with any DPN pharmacologic treatment. The models identifying factors associated with DPN related opioid use and first line opioid use were estimated for only those that had any DPN pharmacologic treatment and excluded those without any use. The chronic use models were only estimated for opioid users. Estimates of the Odds Ratios (OR) and $95 \%$ confidence intervals are reported. All analysis was carried out using SAS version 9.2 (SAS Institute, Cary, North Carolina).

\section{Sensitivity Analyses}

We varied our inclusion criteria in a series of sensitivity analyses that primarily explore the certainty that drugs used to treat DPN were being used to treat DPN. The first sensitivity analysis was further restricted to exclude persons with any pre-index opioid exposure thus diminishing the possibility that opioids were used to manage some other pain. With this cohort we have the greatest confidence that opioid use observed would be to treat DPN and not some other condition. We then progressively relaxed our inclusion criteria to include persons who had other potential uses for opioids (pain diagnoses, surgery and cancer) and these are described in the bottom of figure 1. Multivariable logistic regression models were conducted for each sensitivity analysis cohort for the DPN drug use measures previously described.

\section{Results}

A total of 363,241 diabetic patients were initially identified from the administrative claims and 666 met all the inclusion criteria (Figure 1). Of those that were retained, DPN pharmacologic treatment was observed for 354 (53.15\%) subjects and 288 (43.24\%) had DPN related drug use. (Table 1) Persons treated with a pharmacologic DPN drug were more likely to be from the West, and have diabetes with organ damage ( $\mathrm{p}<0.05)$. Compared to those who received non-opioid DPN pharmacologic treatments, persons prescribed opioids were more likely to have diabetes with organ damage, nephropathy and higher comorbidity 
burden ( $\mathrm{p}<0.05)$ (Table 2). Of those who received DPN pharmacologic treatment, 154 $(53.47 \%$ ) had DPN related opioid use (Table 2). The most common first line agents used for DPN were; opioids $33.33 \%(\mathrm{n}=96)$, antidepressants $26.39 \%(\mathrm{n}=76)$, anticonvulsants $22.57 \%$ $(\mathrm{n}=65)$, NSAIDs $19.09 \%(\mathrm{n}=55)$, and skeletal muscle relaxants $5.21 \%(\mathrm{n}=15)$. FDA approved agents duloxetine $1.04 \%(\mathrm{n}=3)$ and pregabalin $5.56 \%(\mathrm{n}=16)$ were used infrequently (Table 3 ). The most frequently prescribed first line opioids were; hydrocodone combinations $14.93 \%(n=43)$ and oxycodone $6.94 \%(n=20)$ (Table 3). Twenty two percent $(\mathrm{n}=64)$ of DPN patients exclusively used opioids for DPN. The mean treatment initiation time in days for opioids was $48.74 \pm 92.32$ days, while for non-opioids was $88.36 \pm 94.22$ days. Daily dose in Meq for DPN related opioids was $32.33 \pm 33.48$ ( $\mathrm{n}=154)$ meq, non-class 2 opioids $31.29 \pm 24.87(n=115)$, class 2 short acting opioids $34.09 \pm 34.77(n=22)$, and more than 1 opioid class $48.89 \pm 67.96(n=17)$ (Data not shown). Only 3 subjects used opioids chronically.

The results from the multivariate logistic regression showed that subjects from the West $(\mathrm{OR}=1.89,95 \% \mathrm{CI}=1.01-3.53)$, used insulin $(\mathrm{OR}=1.49,95 \% \mathrm{CI}=1.02-2.16)$ were more likely to receive a DPN related drug while subjects who saw an endocrinologist $(\mathrm{OR}=0.45$, $95 \% \mathrm{CI}=0.32-0.82)$ were less likely (Table 4$)$. There was only one factor associated with DPN related opioid use; subjects with their initial DPN diagnosis in 2001-2002 (OR=0.34, $95 \% \mathrm{CI}=0.12-0.99)$. Subjects with organ damage or nephropathy were more likely to receive first line opioid therapy $(\mathrm{p}<0.05)$ (Table 4).

\section{Sensitivity analysis results}

Our first sensitivity analysis that excluded subjects with prior opioid use included 464 subjects and $37.07 \%(n=172), 13.58 \%(n=63)$, and 22.67\% ( $n=39)$ had DPN related drug use, DPN related opioid use, and first line opioid use, respectively. As we relaxed the exclusion criteria and incrementally added the excluded conditions, DPN related drug use and DPN related opioid usage increased; $57.83 \%$ and $26.31 \%$ for the sensitivity analysis that included persons with pain conditions had DPN related drug and DPN related opioid use, respectively, and these same measures were $61.12 \%$ and $32.45 \%$ for the sensitivity analysis that also included subjects with cancer and surgery, and were $70.61 \%$ and $49.29 \%$ for our most liberal cohort of 5,792 subjects that had no pain, surgery, cancer, or prior opioid restrictions. DPN patients with pain diagnosis and disease burden were more likely to receive DPN related opioids, for longer durations and at higher doses in the patients with pain or surgery/cancer groups. Additionally, since the DPN patients in the first three sensitivity analyses did not have pre-index opioid exposure, first line opioid use was not associated with pain diagnoses or the count of pain conditions (Data not shown).

In the sensitivity cohorts with pain, cancer, surgery and prior opioid exposure we consistently found that middle aged and older people were less likely to have DPN related opioids compared to the DPN subjects with age of $18-40$ years $(\mathrm{P}<0.05)$. Also in these groups, Midwest and West regions had higher DPN related opioid use compared to the subjects from the East. The count of pain conditions was strongly related with the DPN related opioid in the sensitivity analysis cohorts with pain, cancer, surgery and prior opioid use conditions. In our most restricted cohort, we observed that females were more likely to 
receive first line opioid use (OR 3.62 CI 1.354-9.659) which is in contrast to our findings from other sensitivity analyses in which females had lower rates of first line opioid use.

\section{Discussion}

This is the first study to explore opioid use in the pharmacologic treatment of DPN. We found that $57 \%$ DPN patients did NOT receive any pharmacologic treatment that could be used for DPN, which is lower than most other studies that have described the rates of pharmacologic treatment of DPN patients. ${ }^{26-27}$ The rates we observed may reflect possible under-treatment or may be due to other factors. Our study did not account for over-thecounter pain medicines or other non-pharmacologic treatment options from the claims data. Additionally, the DPN patients with the most common co-morbidities: low back pain, arthritis/joint pain, depression and anxiety disorders were excluded to define the primary cohort; this rate can be viewed as a lower bound of DPN pharmacologic treatment. Also, the previous studies describing the DPN treatment rates used surveys or cross-sectional data to estimate the DPN prevalence and treatments which might also explain the higher DPN pharmacologic treatment rates than our rates which followed persons for one year after their initial DPN diagnosis.

Despite the neuropathy treatment guidelines that discourage use of opioids as first line treatment, opioids were used by over $50 \%$ of persons receiving a DPN pharmacologic treatment and opioids were the most frequently prescribed first line agents and is consistent with a recent study, that showed that opioids were used as first line agents in $28 \%$ of DPN patients. ${ }^{28}$ This finding was robust across all of our sensitivity analyses. Further, we observed the mean initiation time in days for opioids was almost half compared to nonopioid DPN drugs which further demonstrate prescribers' preference for opioids over nonopioids in DPN. Approximately half of patients prescribed opioids used them exclusively while the remainder used opioids in conjunction with another DPN pharmacologic treatment. Also, all but three patients used opioids on a short term basis ( $<90$ days), so it appears that physicians use opioids as an acute analgesic strategy and frequently initiate opioids without trying alternative guideline recommended therapies to manage DPN at the outset. Duloxetine (2004) and pregabalin (2005) are the only agents approved by the FDA

for the treatment of $\mathrm{DPN}^{29}$; however, these drugs were used infrequently in this sample. On one hand this is concerning because prescribing opioids can lead to misuse, abuse, dependency, tolerance and potentially to increased mortality due to overdose of opioids. ${ }^{30}$ On the other hand, we found that few persons used opioids on a chronic basis. High rates of concurrent opioid use have also been found in DPN patients treated with pregabalin.

Practitioner choice of prescription opioids for non-cancer pain continues to increase posing serious challenges for healthcare practitioners and policy makers. ${ }^{19}$ Currently, about 90 to $95 \%$ of opioids are prescribed for non-cancer pain conditions ${ }^{17}$ (NCPCs). Increases in rates of diagnosis of NCPCs, use of prescription opioids and increased cumulative yearly doses for NCPC patients have also been observed. ${ }^{31}$ Opioids are very potent analgesics, whose abuse and accidental overdose are responsible for many ER visits and deaths ${ }^{30}$ prompting the FDA to develop Risk Evaluation and Mitigation Strategies (REMS) for certain opioid products. ${ }^{32}$ The evidence regarding the use of opioids in managing neuropathic pain 
conditions requires balancing risks and benefits. A recent Cochrane review assessed the efficacy and safety of opioids in the management of neuropathic pain and found that opioids reduced pain more than placebo by was associated with increased adverse effects such as constipation, drowsiness, and nausea and vomiting. ${ }^{33}$ Opioids used in conjunction with nonopioids could achieve better analgesia with lower individual doses but may be offset by opioid related side effects. ${ }^{34}$ These studies provide evidence that opioids can alleviate the pain associated with DPN for those that can tolerate the side effects however the risk benefit profile of opioids used chronically over years is unknown and must be weighed against the increasing risk of opioid misuse and abuse that is a concern when treating any type of pain.

NSAIDs are not considered as drugs of choice in the chronic neuropathic pain conditions including DPN. ${ }^{35-36}$ However, we found high usage of NSAIDs in treating DPN, which is consistent with previous research. ${ }^{37}$ Also, this estimate of NSAIDs use might be understated since we were not able to capture all the over the counter NSAID use. Although we excluded DPN patients with the most common pain or co-morbid conditions, it is still not possible conclusively determine whether this high use of NSAIDs is due to DPN or possibly other comorbidities not excluded. The rates of NSAID use that we observed, which were greater than rates of use for approved pharmacologic DPN treatment is also troubling given the potential bleeding and cardiovascular risk associated with NSAIDs. ${ }^{38-39}$

In our primary cohort, we did not identify many factors prognostic for DPN pharmacologic treatment, DPN related opioid use, or first line opioid use. Insulin use was associated with a higher likelihood of receiving DPN pharmacologic treatment but not opioid use. This may indicate that these patients have severe DPN symptoms and were more likely to receive aggressive pharmacologic management. Similarly, persons with diabetes with complications and those with nephropathy were more likely to use opioids indicating that those with more severe diabetes were more likely to be treated and were more likely to use opioids. However this finding should be interpreted cautiously, with the exception of one sensitivity analysis, these associations were not observed in other sensitivity analyses.

In our sensitivity analyses, which incrementally included DPN patients with excluded conditions, additional factors were associated with DPN related opioid use and first line use. Most notably, the presence of multiple pain conditions and higher baseline disease burden were strongly associated with DPN related opioid use in those sensitivity analyses that included persons with pain conditions. It is possible that clinicians may be prescribing these drugs to manage both the DPN and other painful conditions but without a diagnosis that can be clearly linked to a prescription, this interpretation is only speculative.

We also observed that females were more likely to receive any DPN treatment, but were less likely to receive opioids as a first therapeutic agent for DPN. This may be because females with DPN might have less tolerance for DPN symptoms and more sensitivity towards painful stimuli, leading to higher chances of receiving DPN pharmacologic treatment. ${ }^{40}$

Based on the nature of claims data, this study has the following limitations. First, our final sample only included 666 patients, and of those only 354 had received DPN treatment. As a result, it is unclear if factors that were not found to be significant in our logistic models 
using the primary cohort were truly not associated with opioid use or first line opioid use or there was not adequate power to detect differences. Our sensitivity analyses which were larger identified more factors prognostic for opioid and first line opioid use. Second, availability of other OTC pain medications including topical agents such as capsaicin could not be fully captured and our rates or DPN pharmacologic treatment may be understated. Third, in clinical practice, opioids are used for numerous pain conditions, so linking use of opioids for any particular condition is difficult. We restricted the sample to exclude those with pain conditions that are most often associated with DPN drug use to mitigate this and employed a series of sensitivity analyses that relaxed those restrictions and in turn our confidence that the opioid use was for DPN is incrementally diminished. Fourth, we attempted to identify associations between prescriber type and receipt of DPN drugs and opioid prescribing. The assignment of a prescriber type was based on the frequencies of prescriptions written by each prescriber type and it is not known if this was the physician who ultimately was responsible for deciding upon DPN pharmacologic treatments. Lastly, our findings may be influenced by the different states' Medicaid and commercial insurance formulary policies, which may steer patients to DPN treatments and these could not be captured in our claims data.

\section{Conclusion}

About $57 \%$ of DPN patients remained untreated with pharmacologic therapy a year after an initial DPN diagnosis, which may reflect under-treatment. Despite DPN treatment guidelines that do not recommend opioids as first line treatment, opioids were among the most frequently prescribed first line agents. Given the availability of other agents to manage DPN that do not possess the risks of tolerance and potential abuse, efforts to discourage use of opioids as first line agents should be explored that might include clinical decision support tools, practitioner training, and incorporating multidisciplinary practices with clinical pharmacists. Besides persons with diabetic complications, there were no consistent factors associated with opioid use making it difficult to target patient or provider groups.

\section{Supplementary Material}

Refer to Web version on PubMed Central for supplementary material.

\section{Acknowledgments}

Data acquisition was supported by the University of Arkansas Translational Research Institute (CTSA grant \# UL1TR000039). We would like to thank Gary Moore for his programming assistance in preparing the analytic research file.

\section{Reference List}

1. Tesfaye S, Stevens LK, Stephenson JM, et al. Prevalence of diabetic peripheral neuropathy and its relation to glycaemic control and potential risk factors: the EURODIAB IDDM Complications Study. Diabetologia. 1996; 39:1377-1384. [PubMed: 8933008]

2. Gordois A, Scuffham P, Shearer A, et al. The health care costs of diabetic peripheral neuropathy in the US. Diabetes Care. 2003; 26:1790-1795. [PubMed: 12766111] 
3. Dyck PJ, Kratz KM, Karnes JL, et al. The prevalence by staged severity of various types of diabetic neuropathy, retinopathy, and nephropathy in a population-based cohort: the Rochester Diabetic Neuropathy Study. Neurology. 1993; 43:817-824. [PubMed: 8469345]

4. Sadosky A, McDermott AM, Brandenburg NA, et al. A review of the epidemiology of painful diabetic peripheral neuropathy, postherpetic neuralgia, and less commonly studied neuropathic pain conditions. Pain Pract. 2008; 8:45-56. [PubMed: 18211592]

5. Alvarsson A, Sandgren B, Wendel C, et al. A retrospective analysis of amputation rates in diabetic patients: can lower extremity amputations be further prevented? Cardiovasc Diabetol. 2012; 11:18. [PubMed: 22385577]

6. Aragon-Sanchez J, Lazaro-Martinez JL, Pulido-Duque J, et al. From the diabetic foot ulcer and beyond: how do foot infections spread in patients with diabetes? Diabet Foot Ankle. 2012; 3

7. Daousi C, MacFarlane IA, Woodward A, et al. Chronic painful peripheral neuropathy in an urban community: a controlled comparison of people with and without diabetes. Diabet Med. 2004; 21:976-982. [PubMed: 15317601]

8. O'Connor AB, Dworkin RH. Treatment of neuropathic pain: an overview of recent guidelines. Am J Med. 2009; 122:S22-S32. [PubMed: 19801049]

9. Tesfaye S, Boulton AJ, Dyck PJ, et al. Diabetic neuropathies: update on definitions, diagnostic criteria, estimation of severity, and treatments. Diabetes Care. 2010; 33:2285-2293. [PubMed: 20876709]

10. Habib AA, Brannagan TH III. Therapeutic strategies for diabetic neuropathy. Curr Neurol Neurosci Rep. 2010; 10:92-100. [PubMed: 20425233]

11. Attal N, Cruccu G, Haanpaa M, et al. EFNS guidelines on pharmacological treatment of neuropathic pain. Eur J Neurol. 2006; 13:1153-1169. [PubMed: 17038030]

12. Attal N, Cruccu G, Baron R, et al. EFNS guidelines on the pharmacological treatment of neuropathic pain: 2010 revision. Eur J Neurol. 2010; 17:1113-1e88. [PubMed: 20402746]

13. Boulton AJ. Guidelines for diagnosis and outpatient management of diabetic peripheral neuropathy European Association for the Study of Diabetes, Neurodiab. Diabetes Metab. 1998; 24(Suppl 3): 55-65. [PubMed: 9881234]

14. Boulton AJ, Vinik AI, Arezzo JC, et al. Diabetic neuropathies: a statement by the American Diabetes Association. Diabetes Care. 2005; 28:956-962. [PubMed: 15793206]

15. Dworkin RH, O'Connor AB, Audette J, et al. Recommendations for the pharmacological management of neuropathic pain: an overview and literature update. Mayo Clin Proc. 2010; 85:S3-14. [PubMed: 20194146]

16. Moulin DE, Clark AJ, Gilron I, et al. Pharmacological management of chronic neuropathic pain consensus statement and guidelines from the Canadian Pain Society. Pain Res Manag. 2007; 12:13-21. [PubMed: 17372630]

17. Hudson TJ, Edlund MJ, Steffick DE, et al. Epidemiology of regular prescribed opioid use: results from a national, population-based survey. J Pain Symptom Manage. 2008; 36:280-288. [PubMed: 18619768]

18. Richardson LP, Fan MY, McCarty CA, et al. Trends in the prescription of opioids for adolescents with non-cancer pain. Gen Hosp Psychiatry. 2011; 33:423-428. [PubMed: 21749839]

19. Sullivan MD, Edlund MJ, Fan MY, et al. Trends in use of opioids for non-cancer pain conditions 2000-2005 in commercial and Medicaid insurance plans: the TROUP study. Pain. 2008; 138:440 449. [PubMed: 18547726]

20. Fillingim RB, King CD, Ribeiro-Dasilva MC, et al. Sex, gender, and pain: a review of recent clinical and experimental findings. J Pain. 2009; 10:447-485. [PubMed: 19411059]

21. Le TK, Able SL, Lage MJ. Resource use among patients with diabetes, diabetic neuropathy, or diabetes with depression. Cost Eff Resour Alloc. 2006; 4:18. [PubMed: 17059602]

22. Hartsfield CL, Korner EJ, Ellis JL, et al. Painful diabetic peripheral neuropathy in a managed care setting: patient identification, prevalence estimates, and pharmacy utilization patterns. Popul Health Manag. 2008; 11:317-328. [PubMed: 19108647]

23. Charlson ME, Pompei P, Ales KL, et al. A new method of classifying prognostic comorbidity in longitudinal studies: development and validation. J Chronic Dis. 1987; 40:373-383. [PubMed: 3558716] 
24. [Assessed november 23, 2010] Clinical Classification Software Categories for ICD-9 Codes. Available at: http://www.hcup-us.ahrq.gov/toolsoftware/ccs/AppendixAsingleDX..txt

25. Edlund MJ, Martin BC, Fan MY, et al. Risks for opioid abuse and dependence among recipients of chronic opioid therapy: results from the TROUP study. Drug Alcohol Depend. 2010; 112:90-98. [PubMed: 20634006]

26. Charles M, Ejskjaer N, Witte DR, et al. Prevalence of neuropathy and peripheral arterial disease and the impact of treatment in people with screen-detected type 2 diabetes: the ADDITIONDenmark study. Diabetes Care. 2011; 34:2244-2249. [PubMed: 21816977]

27. Tolle T, Xu X, Sadosky AB. Painful diabetic neuropathy: a cross-sectional survey of health state impairment and treatment patterns. J Diabetes Complications. 2006; 20:26-33. [PubMed: 16389164]

28. Kozma CM, Benson C, Slaton TL, et al. Opioids before and after initiation of pregabalin in patients with diabetic peripheral neuropathy. Curr Med Res Opin. 2012; 28:1485-1496. [PubMed: 22856535]

29. Veves A, Backonja M, Malik RA. Painful diabetic neuropathy: epidemiology, natural history, early diagnosis, and treatment options. Pain Med. 2008; 9:660-674. [PubMed: 18828198]

30. Paulozzi LJ, Budnitz DS, Xi Y. Increasing deaths from opioid analgesics in the United States. Pharmacoepidemiol Drug Saf. 2006; 15:618-627. [PubMed: 16862602]

31. Sullivan MD, Edlund MJ, Fan MY, et al. Risks for possible and probable opioid misuse among recipients of chronic opioid therapy in commercial and medicaid insurance plans: The TROUP Study. Pain. 2010; 150:332-339. [PubMed: 20554392]

32. [Assessed December 5, 2010] The Food Drug Administration (FDA) - Opioid Drugs and Risk Evaluation and Mitigation Strategies (REMS). Sep 1. 2009 Available at: http://www.fda.gov/ Drugs/DrugsSafety/InformationbyDrugClass/ucm163647.htm

33. McNicol ED, Midbari A, Eisenberg E. Opioids for neuropathic pain. Cochrane Database Syst Rev. 2013; 8:CD006146. [PubMed: 23986501]

34. Gilron I, Bailey JM, Tu D, et al. Morphine, gabapentin, or their combination for neuropathic pain. N Engl J Med. 2005; 352:1324-1334. [PubMed: 15800228]

35. Wu N, Chen S, Boulanger L, et al. Duloxetine compliance and its association with healthcare costs among patients with diabetic peripheral neuropathic pain. J Med Econ. 2009; 12:192-202. [PubMed: 19705975]

36. Zhao Y, Wu N, Chen S, et al. Changes in opioid use and healthcare costs among U.S. patients with diabetic peripheral neuropathic pain treated with duloxetine compared with other therapies. Curr Med Res Opin. 2010; 26:2147-2156. [PubMed: 20662557]

37. Boulanger L, Zhao Y, Foster TS, et al. Impact of comorbid depression or anxiety on patterns of treatment and economic outcomes among patients with diabetic peripheral neuropathic pain. Curr Med Res Opin. 2009; 25:1763-1773. [PubMed: 19505204]

38. [Assessed April 22, 2013] The Food Drug Adminstration (FDA): Public health advisory- FDA announces important changes and additional warnings for COX-2 selective and conventional nonsteroidal antiinflammatory drugs (NSAIDs). Apr 7. 2005 Available at: http://www.fda.gov/ Drugs/DrugSafety/PostmarketDrugSafetyInformationforPatientand\%20Providers/ucm150314.htm

39. Howard RL, Avery AJ, Slavenburg S, et al. Which drugs cause preventable admissions to hospital? A systematic review. Br J Clin Pharmacol. 2007; 63:136-147. [PubMed: 16803468]

40. Riley JL III, Robinson ME, Wise EA, et al. Sex differences in the perception of noxious experimental stimuli: a meta-analysis. Pain. 1998; 74:181-187. [PubMed: 9520232] 


\section{Key Point}

- Opioids were the most frequently prescribed first line drugs for DPN

\section{Funding agency}

- Data acquisition was supported by the University of Arkansas Translational Research Institute (CTSA grant \# UL1TR000039).

\section{Prior postings and presentations}

- Preliminary results from the study were presented at the International Society for Pharmacoeconomics and Outcomes Research (ISPOR) $17^{\text {th }}$ Annual International Meeting June 2012 Washington, DC, USA. 


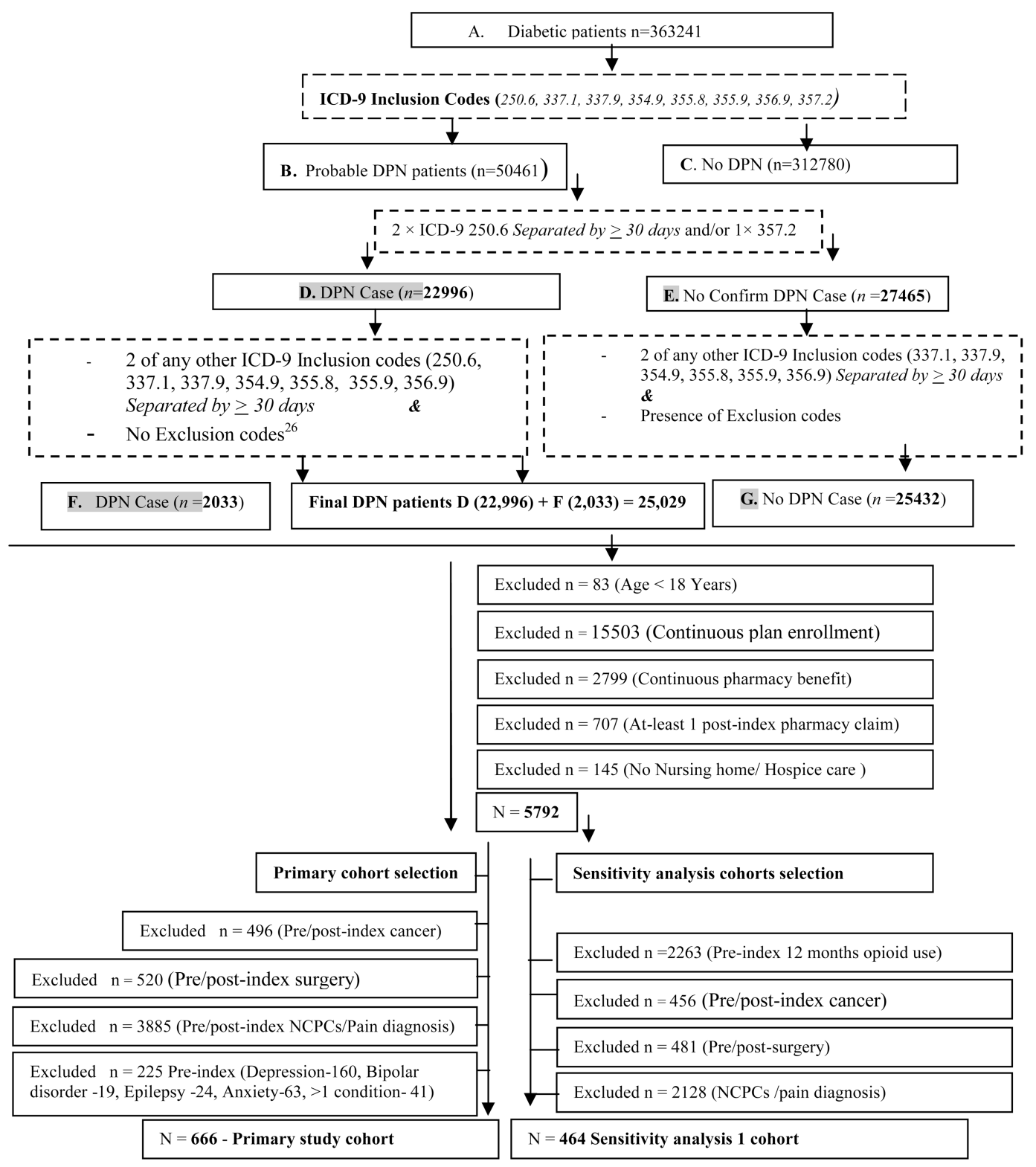

Figure 1. DPN identification ${ }^{22}$, exclusion criteria and selection of primary and sensitivity analysis cohorts 


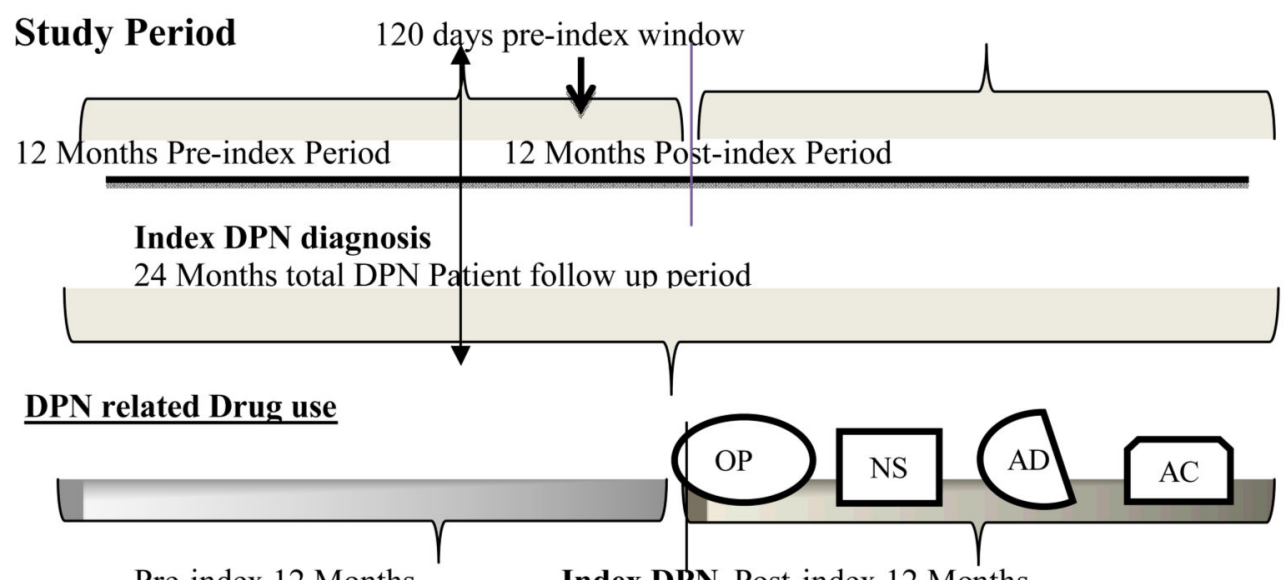

Pre-index 12 Months

Index DPN Post-index 12 Months
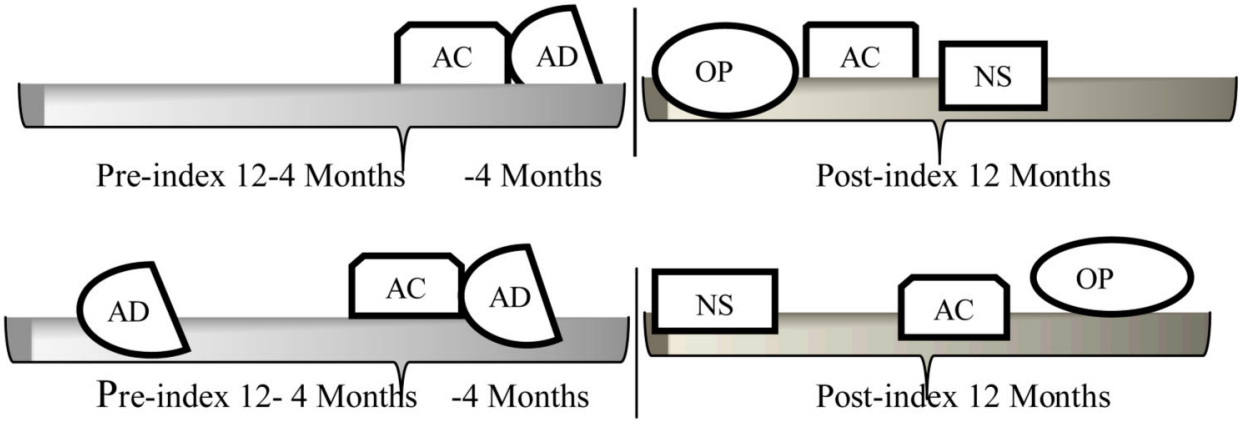

Non DPN related drug use

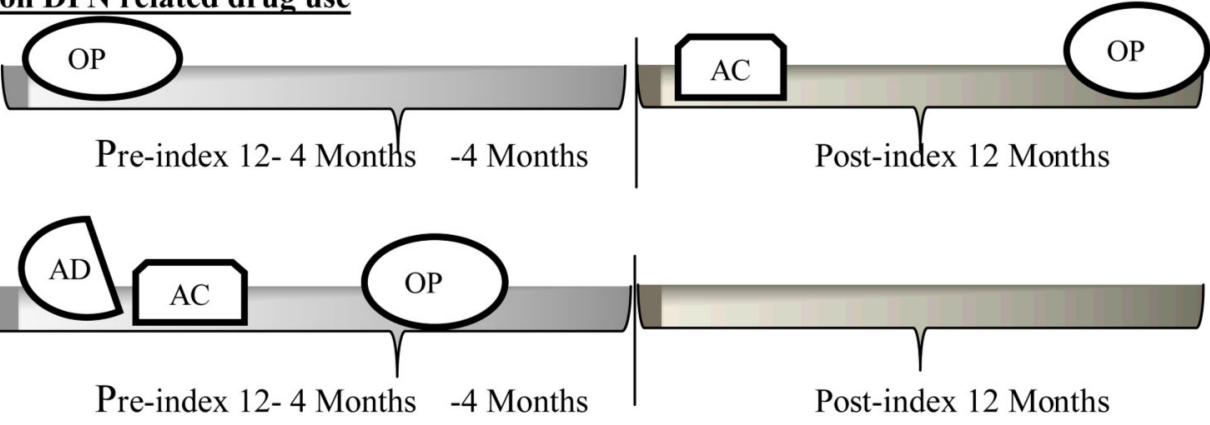

Figure 2. Study period \& description of DPN related and first line DPN drug use 